\title{
Expert Talk for Time Machine Session: Affective Multimedia Analysis: Introduction, Background and Perspectives
}

\author{
Mohammad Soleymani \\ Computer Science Department \\ University of Geneva, Switzerland \\ mohammad.soleymani@unige.ch
}

\begin{abstract}
The term "affective computing" was coined by Rosalind Picard in 1995. She presented her ideas about how to use affect for interaction with and analysis of multimedia. Her ideas were inspiring to the studies and applications on affective multimedia analysis in the last decade. In this talk, the initial ideas and their development to the current state as well as challenges and perspectives are presented.
\end{abstract}

Keywords-affective computing; multimedia analysis; human factors;

\section{SUMMARY}

In 1995, Picard presented her ideas on how to use affective computing for multimedia selection and summarization [1]. The technical paper, which was never accepted as a peerreviewed article at a time, has been inspiring to the field which is, today, known by the report's title, "Affective Computing". Among her ideas, she envisaged a content player which can sense user's emotional state and deliver the content that matches his/her emotional state. Such an application also needs an emotional understanding of the content itself. She also envisioned a video affective content summarization which highlights the most important moments of a video to be watched. In 2001, Hanjalic and $\mathrm{Xu}$ proposed a user oriented affective video characterization which pioneered the track of research which aimed at understanding the affective content of videos using Multimedia Content Analysis (MCA) [2].

With the current rate of the expansion of user generated content. The classic, cognitive indexing methods are showing their limits. Affective indexing is a complementary alternative which attracts multimedia researchers. Users are also expecting content recommendation and delivery systems that can better adapt to their taste and emotions. Although the user interaction and social information is bridging the existing gap between the users and machines, emotional understanding from the content and users will certainly improve users' experience.

Affective computing now has its own journal, IEEE Transactions on Affective Computing, and its biannual conference, Affective Computing and Intelligent Interactions (ACII), however, multimedia community, interested in content analysis, does not have a strong presence in those publications. Multimedia related affective research is being published in different venues and lacks coherence and standardization. Unlike emotion recognition studies which have large number of publicly available databases and challenges. There is a lack of standard benchmarks for video affective analysis. This is partly due to the usage of copyrighted material which prohibits publishing and sharing the datasets. The other reason behind this lack of consensus is that this track of research lacks its own forum which brings together the interested scholars or industrial key players. In this talk, I will present the origins of the idea of using affect in content delivery system, from Picard's technical report and follow its development in the last decade to its current state of the art. The current challenges on this topic includes but not limited to corpus development, identifying the best features and machine learning methods, emotion reporting tools, and ground truth generation.

The focus of the talk will be on affective characterization using MCA and not on affect sensing. Finally, I will give recommendations on corpora development and present examples of public affective corpora which took advantage of trained annotators and crowdworkers, i.e., Mediaeval benchmarking campaign [3], [4].

\section{ACKNOWLEDGMENT}

This work is supported in part by European FP7 Marie Curie Intra-European Fellowship: Emotional continuous tagging using spontaneous behavior (EmoTag).

\section{REFERENCES}

[1] R. W. Picard, "Affective computing," Tech. Report: 321, MIT Media Lab, Tech. Rep., 1995. [Online]. Available: http://affect. media.mit.edu/pdfs/95.picard.pdf

[2] A. Hanjalic and L.-Q. Xu, "User-oriented affective video content analysis," in IEEE Workshop on Content-based Access of Image and Video Libraries (CBAIVL'01), Washington, DC, USA, 2001, pp. 50-57.

[3] C-H. Demarty, C. Penet, G. Gravier and M. Soleymani, "The Mediaeval 2011 affect task: Violent scene detection in Hollywood movies," Mediaeval workshop, Pisa, Italy, 2011.

[4] M. Soleymani and M. Larson, "Crowdsourcing for Affective Annotation of Video: Development of a Viewer-reported Boredom Corpus," in Workshop on Crowdsourcing for Search Evaluation, SIGIR 2010, Geneva, Switzerland, July 2010. 